\title{
Fluorescence enhanced BA-LIFT for single cell detection and isolation
}

\author{
A. Marquez ${ }^{1 *}$, M. Gomez-Fontela ${ }^{1,2^{*}}$, S. Lauzurica ${ }^{1}$, R. Candorcio ${ }^{1}$, D. Munoz- \\ Martin $^{1}$, M. Morales ${ }^{1}$, M. Ubago ${ }^{1}$, C. Toledo ${ }^{1}$, P. Lauzurica ${ }^{2}$, C. Molpeceres ${ }^{1}$ \\ ${ }^{1}$ Centro Láser, Universidad Politécnica de Madrid, Madrid, España \\ ${ }^{2}$ Unidad de Activación Inmunológica, Centro Nacional de Microbiología, Instituto de Salud Carlos III, \\ Madrid, España. \\ ${ }^{*}$ Both authors have contributed equally to this article.
}

E-mail: andres.marquez@upm.es

Received xxxxxx

Accepted for publication $\mathrm{xxxxxx}$

Published $\operatorname{xxxxxx}$

\section{Abstract}

Laser bioprinting is a term that refers to a group of laser-based techniques for printing living cells with high precision and good viability. Most of these techniques are based on modifications of the standard Laser Induced Forward Transfer Technique (LIFT). When it comes to printing living material, direct laser irradiation should be avoided, therefore an indirect LIFT technique comprising an energy absorption layer should be used. This work presents a blister actuated LIFT (BA-LIFT) technique which uses a commercial polyimide tape as a uniform energy absorption layer. To increase the potential of the technique for cell selection and printing accuracy, we take advantage of the high optical transmission of the polyimide layer to implement an in-line fluorescence and conventional imaging vision system coaxial with the laser path. With this system and using the appropriate staining methodology it is possible to track and identify different cell types, selecting those to be transferred and tracking cell survival both in the donor and acceptor substrates. We studied the BA-LIFT printability map for sodium alginate and methylcellulose hydrogels in the fluence range from $6.1 \mathrm{~J} / \mathrm{cm}^{2}$ to $2.0 \mathrm{~J} / \mathrm{cm}^{2}$ together with the cell viability assessment measured by our fluorescence system. The study has revealed that less concentrated, therefore less viscose hydrogel shows better results with lower fluences, whereas hydrogels with higher concentrations present better results at higher fluences. Also, at low fluences $98 \%$ of cell viability was obtained, besides both primary cells and cell lines keep their integrity, proliferating and functional activity. The technique was tested by tracking and targeting mouse hematopoietic progenitor stem cells transferred to assess colony forming units; moreover, natural killer cells were isolated and its activation in a stimulation media was tracked with the fluorescence system.

Keywords: Laser Induced Forward Transfer, BA-LIFT, bioprinting, single cell isolation, fluorescence microscopy, colony forming units, natural killer cell activation

\section{Introduction}


applications use an approach based on a set-up comprising a transparent donor substrate coated with an energy absorption layer and the material to be printed. Laser pulses are absorbed by the intermediate layer inducing the formation of a jet, transferring droplets of the biomaterial from the donor towards the acceptor substrate placed in parallel and separated at a certain gap distance [9]. For biological applications, like cell printing, the energy absorption layer can be made of a thin metallic layer (titanium, titanium oxide, silver or gold) [6,7,10-13], a hydrogel layer [14-16] or a polyimide layer [17-19]. On the last approach the laser pulse is absorbed by the substrate and polyimide interface, vaporizing a part of the polymer layer and creating a vapor bubble that pushes and plastically and elastically deforms the remaining polyimide. The blister formed mechanically pushes the thin liquid layer situated on the polyimide, generating a jet and the corresponding droplet in a process called Blister ActuatedLIFT (BA-LIFT) [17]. The physics of both blister creation and jet droplet formation in BA-LIFT have been widely studied [17,19-22].

Polyimide is a widely used material taking advantage of its excellent structural, electrical and thermal properties for applications that go from the electronics industry to the medical field [23]. Moreover, polyimide possess a great biocompatibility and it has been used as a bio-inert material for implants, sensors and cell culture [24-29].

Polyimides like other polymers have low wettability properties requiring a surface treatment when high wettability is required [23]. Typical surface treatments used are long UV exposure, plasma surface activation or chemical etching.

One of the benefits of using polyimide layers is its transparency to the visible light. This allows to combine standard and fluorescence microscopy in the same optical path for the eventual cell detection and transfer. Fluorescence microscopy has arisen as a powerful tool for cell and molecular biology, allowing the direct visualization of the inner workings of physiological processes, as well as the identification, visualization and tracking of specific cell populations within a complex sample. Having available a great number of fluorescent probes and proteins, high performance detectors with high sensitivity and a great variety of light sources and filters allows the integration of complete systems for faster and more precise fluorescence systems. [30]. The combination of fluorescence imaging, together with the optical properties of the polyimide layers, permits to track cell populations within the sample both before and after the transfer process. Moreover, the use of viability markers allows to track cell viability in situ during the whole process.

Single cell analysis technologies have revealed heterogeneity between individual cells of the same cell type within a tissue. Detail characterization of cellular genome, transcriptome [31], proteome and metabolome [32] enables the high-throughput profiling of cell characteristics that allow to define the functions that will develop both in normal and pathological processes. Although several methods have been developed for the isolation of a single cell [33], there are limitations in terms of the ability to obtain a single cell from complex samples, from cells that do not express certain markers, or the ability to discern the efficiency of isolation. Therefore, BA-LIFT emerges as a promising technique for the isolation of individual cells within a complex sample of previously enriched cell populations, by microfluidic or limiting dilution methods, in order to perform a deeper characterization.

In this work, an original approach for single cell isolation using a BA-LIFT system with a commercial polyimide layer is proposed (Fluorescence enhanced BA-LIFT or FE BALIFT); this layer comes in the form of an adhesive tape which is easily applied onto the slide. The polyimide layer allows the implementation of a fluorescence and conventional microscopy module to enhance the potential of single cell detection and isolation properties of the system. Also, the printability maps for two biocompatible and widely used hydrogels: sodium alginate and methylcellulose are presented, thus providing a general overview of the printing results related to the BA-LIFT parameters. Consequently, these maps help to obtain the optimum parameters in terms of quality and size of the printed droplets. Cell traceability, single cell isolation, and viability has been evaluated using the best transfer conditions, through a combination of fluorescence markers and propidium iodide staining of the cells involved in the assessment. Moreover, the system's biological functionality was proven with 2 applications requiring the selection and detection by fluorescence markers. Firstly, by isolating natural killer (NK) cells individually and in groups to track their activation by transferring them in stimulation media for $6 \mathrm{~h}$. Secondly, by assessing the formation of colonies by transferring fluorescent marked hemopoietic and progenitor stem cells (HPSC's) from the bone marrow of a mouse.

\section{Materials and Methods}

\subsection{Configuration of the BA-LIFT bioprinting device}

The BA-LIFT system comprises a DPSS laser source working at a $355 \mathrm{~nm}$ wavelength (Crylas FTSS355-Q4) emitting $1.3 \mathrm{~ns}$ pulses at a rate of $1000 \mathrm{~Hz}$. The pulse energy is controlled by means of a half-wave plate and a beam polarizer ranging from $1 \mu \mathrm{J}$ to $23 \mu \mathrm{J}$. The laser beam is focused onto the sample using a $10 \mathrm{X}$ microscope objective. The system is equipped with an X-Y servo positioning system and with a manual vertical movement table to place the samples on the focal plane of the vision system and the laser.

A fluorescence setup is implemented to the laser system to monitor the results simultaneously to the transfer process 


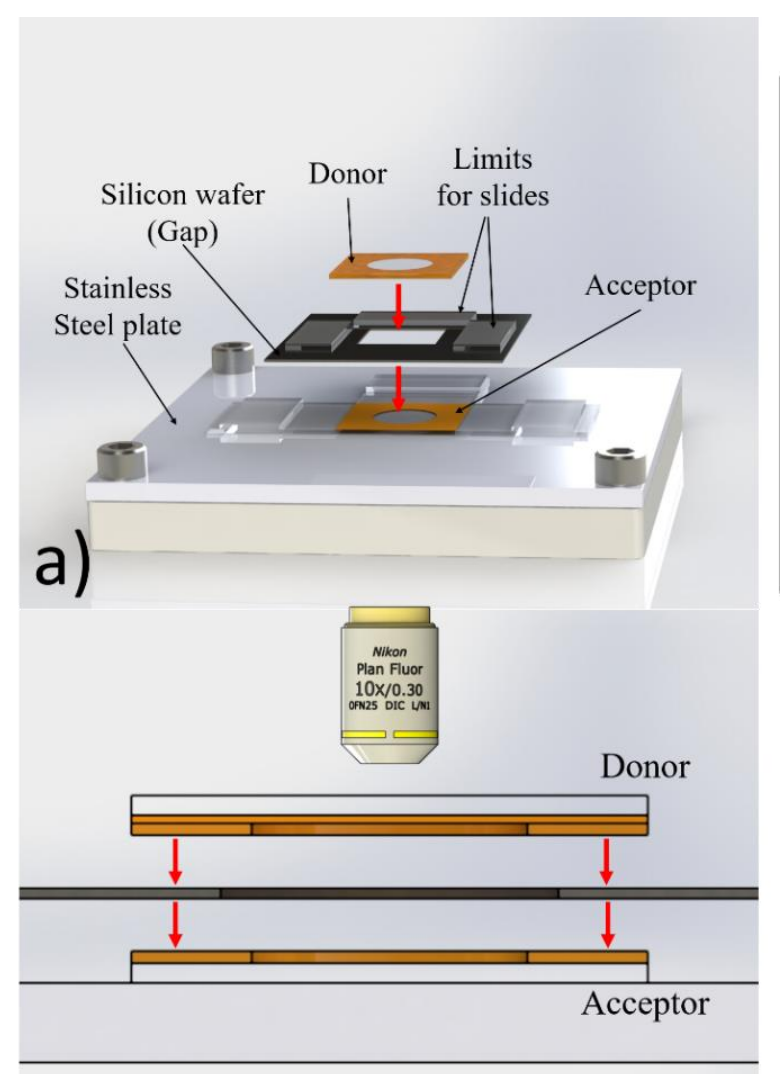

b)

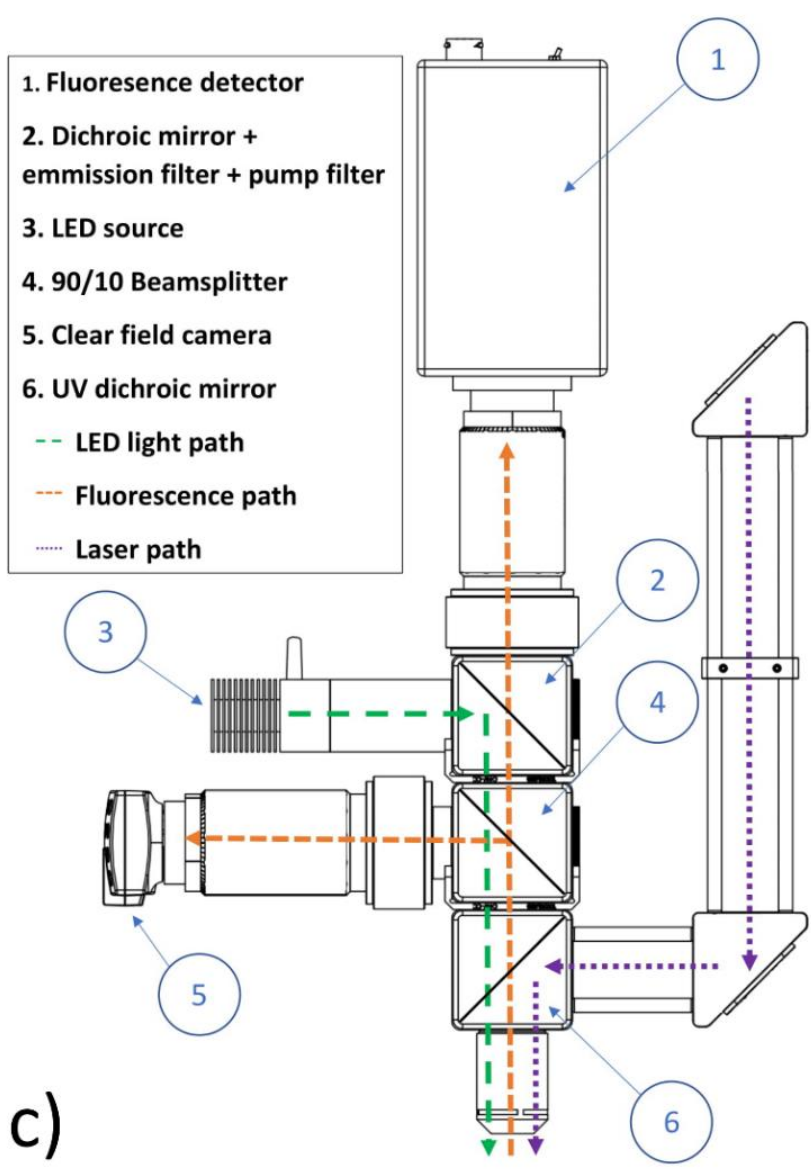

c)

Figure 1. Scheme of device used for FE BA-LIFT. a) 3D representation of how the device used for FE BA-LIFT experiments is assembled; $b$ ) $2 D$ section representation of assembly used in FE BA-LIFT showing the wells made of polyimide; c) Schematic of FE BA-LIFT showing all elements included in the setup together with all optical paths followed by the light, fluorescence and laser.

(Figure 1c). The system consists of a LED source pumping in the required wavelength (Figure 1c ' 3 '). This light is filtered (Figure 1c '2') (excitation filter) so that the wavelength needed to excite the respective fluorophore remains. Filtered light goes through a set of dichroic mirrors allowing the transmittance of visible light but act as mirrors for UV light. This set-up permits to focus the laser beam through the same microscope objective used by the imaging system. The signal obtained by the sample goes first through a beam splitter (Figure 1c '4') dividing the light in two paths; $10 \%$ of the light goes to the clear field image camera (Figure 1c '5') and the remaining $90 \%$ passes through another filter (Figure 1c '2') (emission filter) before reaching the fluorescence detector (Figure 1c '1').

\subsection{Sample preparation and characterization}

BA-LIFT is performed by printing material from a transparent substrate called donor to another substrate called acceptor. Substrates are kept separated by a small gap $(590 \mu \mathrm{m}$ unless specified differently) and fixed together through the assembly shown in Figure 1. Both donor and acceptor are made of a $25 \mathrm{~mm}$ x $25 \mathrm{~mm}$ soda lime microscope slide.

\section{Donor and Acceptor slides}

On the donor a layer of thin polyimide tape (Caplinq PIT0.5S-UT/19) is applied, working as the energy absorption layer; here is where the blister is formed. Afterwards a second and thicker layer $(\sim 70 \mu \mathrm{m})$ of polyimide tape is applied on top of the previous layer (Caplinq PIT1S/19), subsequently a 14 $\mathrm{mm}$ diameter circle is cut on this layer and then removed, creating a well that helps contain the hydrogel with the expected thickness to be coated afterwards. The acceptor is coated only with one thick polyimide tape (Caplinq PIT1S/19), and a $14 \mathrm{~mm}$ diameter circular is also cut and removed. Both donor and acceptor are thoroughly cleaned before use and assembled as shown in Figure 1. In detail, the acceptor is placed on top of a stainless-steel plate, afterwards a $520 \mu \mathrm{m}$ silicon wafer is placed on top of the acceptor acting as a gap between acceptor and donor. Finally, $15 \mu \mathrm{l}$ of the hydrogel is placed on the donor and spread with a blade coater; immediately after, the slide is turned over and placed on top of the silicon wafer facing the acceptor slide. This configuration seals the environment between both slides, keeping a humid environment on the donor and acceptor, preventing the deposited droplets to dry, making it easier to 
take images of the acceptor after the process (Figure 1a). All elements are kept in place thanks to some limits attached to the stainless steel plate and the silicon wafer (Figure 1a).

\section{Plasma surface activation}

To improve wettability properties of the polyimide layer, plasma surface activation is performed exposing its surface to a plasma electrically generated by microwave irradiation. In this process, the samples are placed inside of a small glass desiccator chamber connected to a vacuum pump and taken to a pressure of approximately $133 \mathrm{~Pa}$. The desiccator is exposed to microwave radiation until plasma forms and ionizes the surface.

\section{Hydrogel preparation}

Cell culture media (Lonza RPMI 1640 or GIBCO IMDM) is used for the preparation of the hydrogel layer. Sodium alginate (Sigma-Aldrich) is dissolved in a saline solution $(\mathrm{NaCl} 0.9 \%)$ to a mass concentration of $4 \%$; afterwards it is mixed with RPMI 1640 to obtain the final mass concentrations of $2.0 \%$ and $1.5 \%$. Methylcellulose (R\&D Systems) is mixed with IMDM to the final mass concentrations of $1.5 \%, 1.2 \%$, $1.0 \%, 0.8 \%, 0.5 \%$ and $0.3 \%$. All solutions are stored at $4{ }^{\circ} \mathrm{C}$ and left at ambient temperature before being used.

\section{Cell lines, viability measurement and proliferation assays}

A B-lymphocyte cell line that constitutively express $e G F P$ (C1R-N1-85) and a T-lymphocyte cell line (Jurkat) were used. Staining with a phycoerythrin conjugated monoclonal antibody against CD45 ( $\alpha$-CD45/PE, Biolegend, 368510) was performed as follows: $1 \times 10^{6}$ Jurkat cells were stained with $0.05 \mu \mathrm{g}(2 \mu \mathrm{l})$ of $\alpha$-CD $45 / \mathrm{PE}$ for 30 minutes at $4^{\circ} \mathrm{C}$ in dark conditions, and then washed two times with PBS1X. Finally, cells were mixed with the hydrogel to obtain concentrations of $8-10 \times 10^{6}$ cells per milliliter and extended over the donor layer, allowing to visualize them by the camera set-up.

For viability test, the printed cells were stained with propidium iodide (PI), a DNA intercalating agent used to discriminate between viable and non-viable cells, both in the donor and in the acceptor layer. Fluorescent cells were labeled as dead. Proliferation was assessed by means of optical microscopy and tracking cells every day during a period of 4 days.

\section{Cell activation assays}

A human natural killer (NK) cell line which express an IFN $\gamma$-eGFP fusion protein was used to perform cell activation assays (unpublished data). Cells were transferred to wells of a 96 well culture plates containing a stimulation media (50ng PMA and $1 \mu \mathrm{g}$ ionomycin). A modified plate was used to place the donors on top for keeping a sealed environment and sterile conditions during the transfer (4 mm gap). Cells were incubated for 6 hours after the transference and IFN $\gamma$-eGFP production was assessed by fluorescence microscopy.

\section{HSPC's isolation and Colony Forming Units (CFU) assay}

Mouse bone marrow cells were obtained from C57BL6 mice and stained with a biotin conjugated antibody cocktail consisting on anti-CD3, anti-CD4, anti-CD8, anti-CD19, antiCD220, anti-NKp46, anti-CD11b and anti-CD11c. Cells were then incubated with streptavidin-PE in order to label Lin+ cells as red cells, and Lin- cells as non-fluorescent cells. For HSPC isolation, Lin- cells were BA-LIFT-transferred onto a culture dish with $1.27 \%$ methylcellulose (R\&D Systems, HSC008) and placed in a $37^{\circ} \mathrm{C}$ and $5 \% \mathrm{CO}_{2}$ incubator. A modified lid to place the donors was used to keep a sealed environment and sterile conditions during the transfer $(3 \mathrm{~mm}$ gap). Colonies, which reflected the number of colony forming units (CFU) were counted at different days after the LIFT process.

\section{Results}

\subsection{Characterization of donor substrate.}

In this work a commercial polyimide adherent tape was used for the energy absorption layer, taking advantage of the easy application and thickness uniformity of the commercial film. For all experiments the thinnest available tape was used $(\sim 30 \mu \mathrm{m})$. In the transmittance spectrum shown in Figure 2a it is shown that in the donor, radiation with wavelengths from $300 \mathrm{~nm}$ up to $400 \mathrm{~nm}$ (UV region) is absorbed completely. For longer wavelengths, the layers on the donor become semitransparent letting light pass through. The laser wavelength used for the experiments $(355 \mathrm{~nm})$ is within this absorption region thus, being all absorbed before reaching the hydrogel. In Figure $2 b$ is shown the theoretical heating of the nearest layer of the hydrogel to the polyimide calculated using numerical analysis and calculated using the software package $\mathrm{Comsol}^{\circledR}$. The geometry used for the model mimics the one used in the donor consisting of three different layers; the first is a layer of soda lime glass ( $1 \mathrm{~mm}$ thick), the second a layer of polyimide $(30 \mu \mathrm{m})$ and the third is a layer of water (simulating the hydrogel). Initial temperature is considered as ambient temperature $293.15 \mathrm{~K}$ or $20^{\circ} \mathrm{C}$ and the laser fluence is of $5.6 \mathrm{~J} / \mathrm{cm}^{2}$, all boundaries in contact with air are considered as open boundaries. An axial symmetry in the model is used to speed up the calculation process. Several assumptions and simplifications are introduced in order to make it easier to compute the results. Firstly, no change in phases are considered for any of the materials; secondly, no deformation of the polyimide is introduced, finally the liquid is not transferred hence, staying unaltered and allowing it to heat freely. The model computes the resulting temperature on the surfaces by using a time dependent heat transfer equation (Equation 1). 


$$
\begin{gathered}
\rho C_{p} \frac{\partial T}{\partial t}+\rho C_{p} u \cdot \nabla T+\nabla \cdot q=Q \\
q=-k \nabla T \\
Q=(1-R) \cdot(1-A) \cdot\left(\frac{2 \cdot E_{p}}{\pi \cdot r_{a}^{2}} \cdot 1.133 \cdot \tau\right) \cdot f_{1}(r) \cdot f_{2}(t) \\
f_{1}(r)=e^{\left(-2 \cdot\left(\frac{r}{r_{a}}\right)^{2}\right)} \\
f_{2}(t)=e^{\left(-2 \cdot\left(\frac{t-2 \tau}{\tau}\right)^{2}\right)}
\end{gathered}
$$

contact angle of $7^{\circ} \pm 2^{\circ}$; beyond this time the contact angle starts to increase (Figure 2c).

Another characteristic to consider of the polyimide is the role it plays in the dynamics of BA-LIFT. Blister expansion is produced in the polyimide and gives the energy to the hydrogel to transfer. To make a complete study (and hence complete printability map) it is important to identify when the biggest blister is obtained. In Figure $2 \mathrm{~d}$ it is shown how the blister height profile changes as the beam diameter changes on the interface between the microscope slide and the polyimide (Blisters measured are the result of a plastic deformation on the polyimide only). At position $\mathrm{P}_{0}\left(\omega_{0}=15 \mu \mathrm{m}\right)$, the biggest blister is obtained, as the sample is moved upwards, beam diameter reduces and fluence increases hence a bigger blister is expected. Nonetheless, results show that the opposite occurs because most of the energy is absorbed due to non-linear effects by the microscope slide before it reaches the polyimide interface. Beyond position $\mathrm{P}_{-250}$ no blister is formed, including position P-510 (data not shown) where the smallest beam diameter is obtained $\left(\omega_{0}=5 \mu \mathrm{m}\right)$. If the sample is moved downwards fluence reduces as the beam diameter increases, expecting the blister size to reduce. As shown in Figure $2 d$ at position $\mathrm{P}_{150}$ the blister is wider and smaller corroborating that at position $\mathrm{P}_{0}$ the biggest blister is generated. A second equivalent position for transfer tests was identified, corresponding to a position where the ratio between lost energy in the microscope slide and the beam diameter result in similar fluences as in position $\mathrm{P}_{0}$. This position would be $\mathrm{P}$. $480\left(\omega_{0}=6 \mu \mathrm{m}\right)$, and at this position no blister is observed due to a plastic deformation. Even though, printing is going to be tested and corroborate if transfer takes places due to an elastic deformation. Furthermore, for all the experiments, after blister expansion only plastic and elastic deformation was observed and no polyimide was removed or transferred.

Taken together, these results show the characterization of the technical parameters that influences the BA-LIFT experimental settings used.

\subsection{Printability map}

To create a printability map the transferability of hydrogels was assessed at distinct range of fluences and hydrogel concentrations; characteristics analyzed were shape, diameter and amount of satellite droplets produced during the transfer. Experiments were carried out in both methylcellulose and sodium alginate. Data was taken by performing a total of 24 linear passes per sample, each pass separated $400 \mu \mathrm{m}$ and made at a speed of $400 \mathrm{~mm} / \mathrm{s}$ (@1000 Hz laser frequency) separating the droplets the same distance on the $\mathrm{X}$ and $\mathrm{Y}$ axis. A total of 18 images per set of laser fluence and hydrogel concentration were taken, and 6 to 8 droplets per image were measured and analyzed. The fluence is varied from the maximum until the limit in which no droplets are deposited. ching of 15 seconds and under a vacuum time of 10 secon Treatment persist for 6 minutes, when the sample still had a 

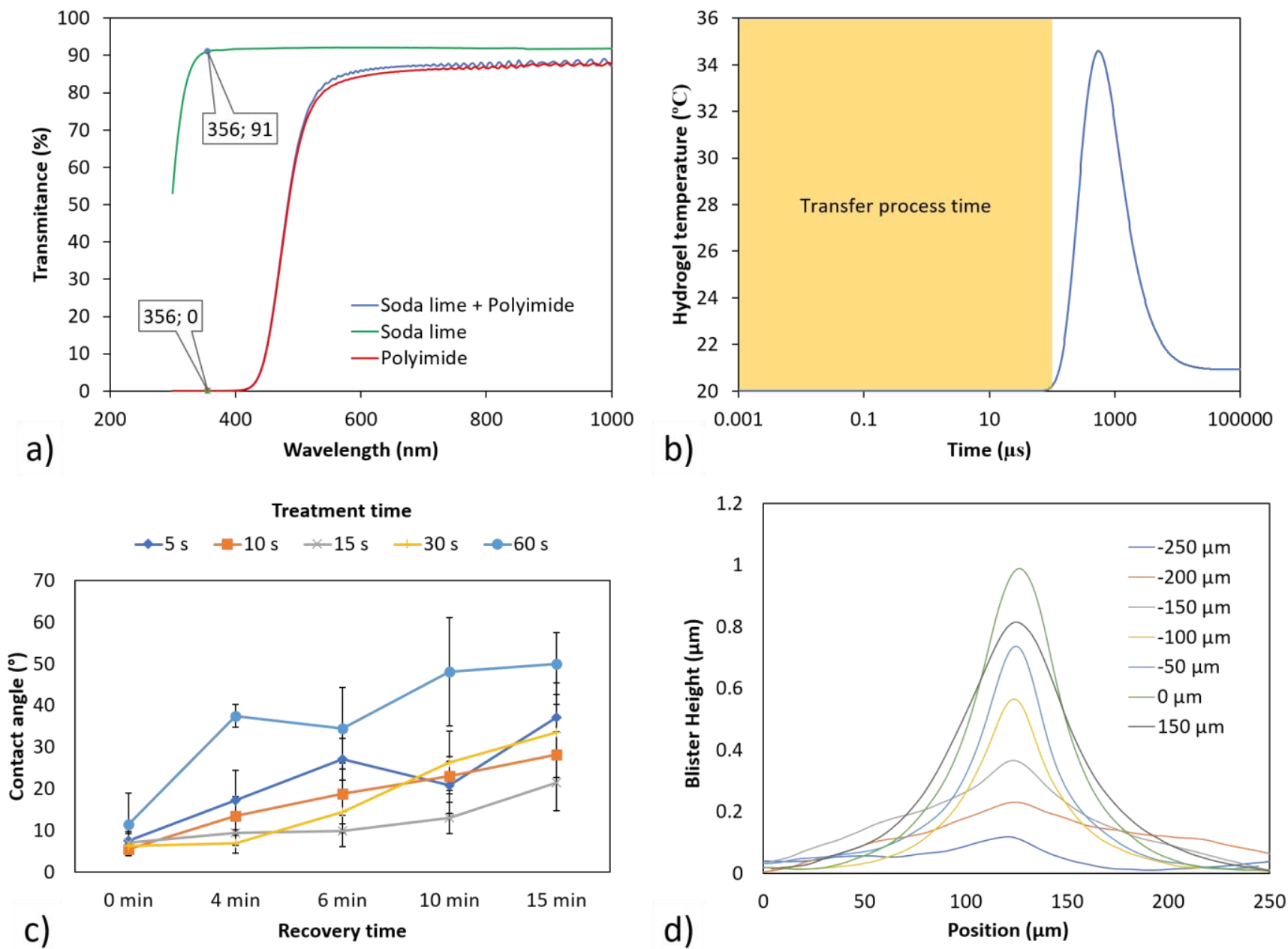

Figure 2. a) Transmittance spectrum of polyimide tape, polyimide tape + soda lime slide and polyimide and polyimide; specific coordinate for $356 \mathrm{~nm}$ shown to emphasize on laser wavelength transmittance; b) Numerical simulation (Comsol ${ }^{\otimes}$ ) of hydrogel heating for a $5.6 \mathrm{~J} / \mathrm{cm}^{2}$ pulse and a $30 \mu \mathrm{m}$ polyimide tape, yellow area indicates typical BA-LIFT droplet transfer time; c) wettability of polyimide tape measured through contact angle at different treatment times and recovery times. d) Blister height profiles at different focal positions of a single 6.0 $\mathrm{J} / \mathrm{cm}^{2}$ pulse.

The printability maps shown in Figure 3a and Figure $3 b$ are presented for both hydrogels showing a summary categorizing the quality of the droplets by a 4-color: red indicates a bad shape with a lot of satellite droplets (Figure 3ci); orange a bad shape with less satellite droplets (Figure 3cii); light green a good shape with some satellite droplets (Figure 3ciii); and dark green good shape without satellite droplets (Figure 3di). Measurement of droplet diameter is made for each set of experiments taking an average of all data and defining the standard deviation as an indicator of repeatability.

Laser fluence used was varied from $(6.5 \pm 0.5) \mathrm{J} / \mathrm{cm}^{2}$ to $(2.3$ $\pm 0.5) \mathrm{J} / \mathrm{cm}^{2}$. Figure $3 \mathrm{c}$ shows the results obtained using sodium alginate at concentrations, $2.0 \%$ and $1.5 \%$. Concentrations higher than $2.0 \%$ don't allow cell growth [35], whereas with concentrations below $1.5 \%$ good results were not achieved. As diameter measurements show a big dispersion and satellite droplets appears in most of the fluence conditions assayed, the parametric window working with this hydrogel is quite narrow.

Like sodium alginate, methylcellulose can be used for cell culture at a mass concentration of $1.5 \%$ as recommended by the manufacturer ( $277.8 \mathrm{mPa} \cdot \mathrm{s}$ viscosity). In comparison with alginate at its maximum accepted concentration (mass concentration of $2.0 \%$ and $30.4 \mathrm{mPa} \cdot \mathrm{s}$ viscosity) methylcellulose has a higher viscosity, requiring higher fluences to be transferred; transfer of $1.5 \%$ methylcellulose droplets was only achieved at the highest fluence. Nevertheless, it is easier to find a trend on data for methylcellulose; at the same transfer fluence droplets are smaller as hydrogel concentration increases (Figure 3d). Also, the same trend is observed when the hydrogel concentration is fixed, and the laser fluence is changed: droplets tend to decrease in size as the fluence reduces. Finally, maximum repeatability is obtained at low fluences where smaller droplets are printed (Figure 3d). 

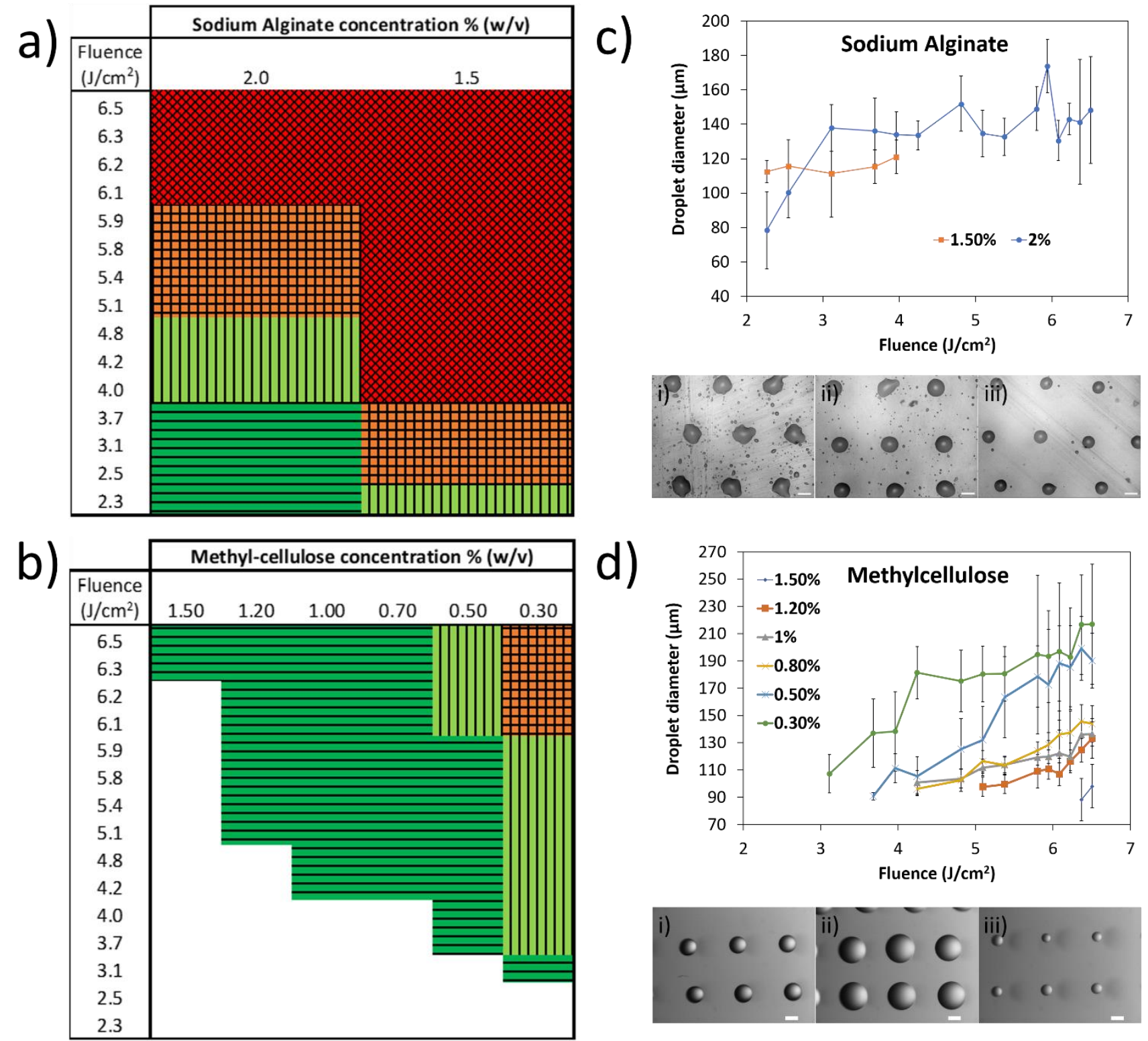

Figure 3. Printability maps results. a) and b) Complete methylcellulose and sodium alginate printability maps respectively. Red (diagonal hatch) indicates a bad shape and with a lot of satellite droplets; orange (square hatch) a bad shape with less satellite droplets; light green (Vertical line hatch) a good shape with some satellite droplets; and dark green (Horizontal line hatch) good shape without satellite droplets. c) Sodium alginate droplet diameters for all experiments. Examples of $2.0 \%$ sodium alginate droplets at: i) $6.1 \mathrm{~J} / \mathrm{cm}^{2}$, ii) 5.1 $\mathrm{J} / \mathrm{cm}^{2}$, iii) $4.8 \mathrm{~J} / \mathrm{cm}^{2}$; d) Examples of methylcellulose droplets at i) $1.0 \%$ @ $6.1 \mathrm{~J} / \mathrm{cm}^{2}$; ii) $0.5 \%$ @ $5.4 \mathrm{~J} / \mathrm{cm}^{2}$; iii) $1.5 \%$ @ $6.4 \mathrm{~J} / \mathrm{cm}^{2}$. (All scale bars $100 \mu \mathrm{m}$ )

Printability results are similar for both positions $\left(\mathrm{P}_{0}\right.$ and $\mathrm{P}$ 480) regarding only size and shape of the droplets (data not shown). Further experiments should be made to understand transfer physical differences between the two working positions. Best results are obtained for methylcellulose in shape, size and repeatability for a wide range of fluences, therefore this hydrogel is proposed to be used for a study of cell printing viability under several conditions.

\subsection{Viability and proliferation}

For viability assays, the same setup as described previously was used, but now mixing the hydrogel with propidium iodide (PI), which allows to identify non-viable cells. Viability of the sample was assessed both before (Figure 5a) and after the printing process (Figure $5 \mathrm{c}$ and d) through a live image (using conventional and fluorescence microscopy), allowing the identification of non-viable or dead cells during the BA-LIFT process. Experiments were carried out using $8-10 \times 10^{6}$ Jurkat and $\mathrm{C} 1 \mathrm{R}$ cells $/ \mathrm{ml}$ in a $0.3 \%$ methylcellulose solution. Viabilities were tested at different fluences and for both work positions described before. Taking advantage of the vision 
system, all cells printed were considered in the counting process and viability calculation. Figure 4a shows viability of cells immediately after the printing process for fluences of 6.1 $\mathrm{J} / \mathrm{cm}^{2}, 5.7 \mathrm{~J} / \mathrm{cm}^{2}, 5.4 \mathrm{~J} / \mathrm{cm}^{2}, 4.8 \mathrm{~J} / \mathrm{cm}^{2}$, and $3.4 \mathrm{~J} / \mathrm{cm}^{2}$ at position $\mathrm{P}_{0}$. At this position no clear tendency can be seen between viability and fluence. Variations up to $6 \%$ in viability between experiments can be seen making it relatively repeatable but not ideal. Maximum viability obtained was of $84 \%$ and a minimum of $68 \%$. Figure $4 \mathrm{a}$ shows results for viabilities immediately after printing for position $\mathrm{P}_{-480}$. Although printing results seem to be similar, there is a clear influence in viability. At all fluences tested viabilities are always higher and with a better repeatability than the other working position. Also, at fluences greater than $2.4 \mathrm{~J} / \mathrm{cm}^{2}$ viability seems not to be further affected by the increase in fluence. The best results were obtained at the lowest fluences with a viability of $98 \%$ and the minimum viability obtained was of $85 \%$. It has been described that the viability of printed cells depends on a combination of different parameters [13]; in our approach cell viability seems not to be greatly affected neither by the hydrogel donor preparation nor by the laser energy, although more experiments are needed to understand the residual cell death inherent to the transfer process in both working positions.

Cell proliferation studies were performed for both Jurkat and C1R cells and at the working position where better results were obtained. Cells were transferred directly into a 96-well plate using a modified lid that allows to prepare the sample within a sterile environment and place the donor on top, keeping it sealed during the transfer process and further cultivation (4 mm gap). Wells were filled with $200 \mu$ l of RPMI culture medium. Proliferation studies were done at fluences of $11 \mathrm{~J} / \mathrm{cm}^{2}, 5.7 \mathrm{~J} / \mathrm{cm}^{2}, 3.1 \mathrm{~J} / \mathrm{cm}^{2}$ and $2.1 \mathrm{~J} / \mathrm{cm}^{2}$. Upper row of Figure $4 \mathrm{~b}$ shows an example of $\mathrm{C} 1 \mathrm{R}$ cells transferred at 2.1 $\mathrm{J} / \mathrm{cm}^{2}$ and cultivated for 4 days; the bottom row is of Jurkat cells transferred at $5.7 \mathrm{~J} / \mathrm{cm}^{2}$ and cultivated during the same time. Proliferation was observed for all fluences up to day 3 , on the $4^{\text {th }}$ day most cells transferred at $11 \mathrm{~J} / \mathrm{cm}^{2}$ were dead; at the rest of the fluences proliferation continued with no problem. The number of cells after culture depends only on the initial number of living cells transferred inside the well and proliferation doesn't seem to be affected by laser fluence.

\subsection{Fluorescence enhanced BA-LIFT applications}

The implementation of a vision system composed of a conventional microscopy and fluorescence modules in the BA-LIFT set-up allows to identify and print cells which express specific surface markers or fluorescent reporters; this feature, in combination with the capability to recognize viable cells before and after the printing process, gives the tools to develop an alternative sorting method to isolate specific cells within a complex population. Furthermore, this system allows to evaluate the survival rate of cells before and after the printer process with a non-toxic staining method.

\section{Single cell isolation}

The same setup used to create the printability map was used to assess the sensitivity of the fluorescence setup for single cell detection and isolation. Two approaches were tested: 1) C1R-N1-85 cell line which constitutively express eGFP (enhanced Green Fluorescence Protein) excitation with a 474 $\mathrm{nm}$ wavelength and emission with $525 \mathrm{~nm}$ wavelength; and 2) Jurkat cell line stained with monoclonal antibodies against surface receptor CD45, labelled with phycoerythrin $(\alpha-$ CD45/PE) excitation with a $531 \mathrm{~nm}$ wavelength and emission with $593 \mathrm{~nm}$ wavelength (Figure 5e and f).

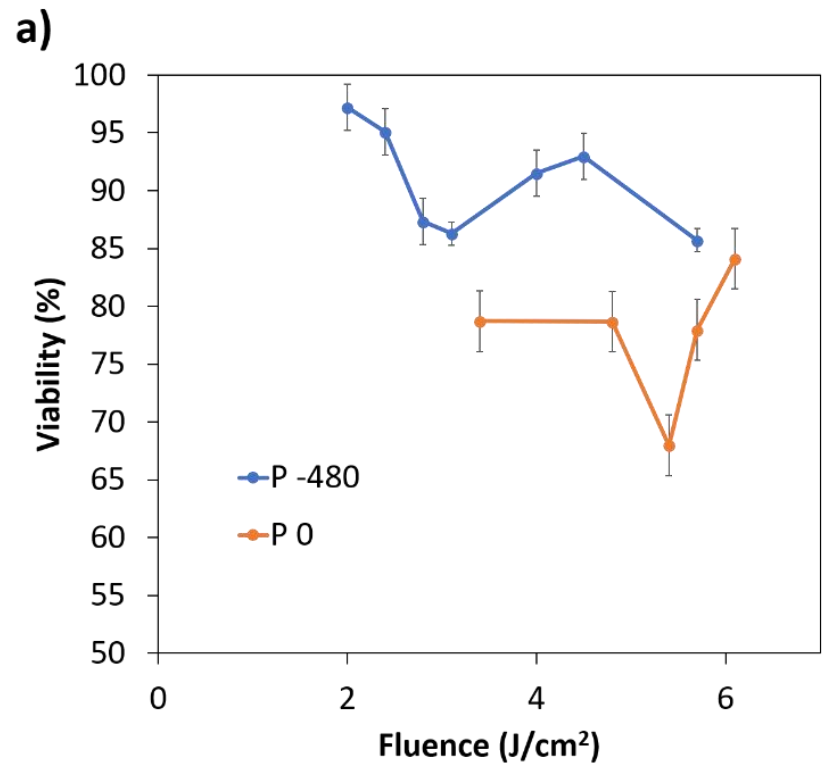

b)

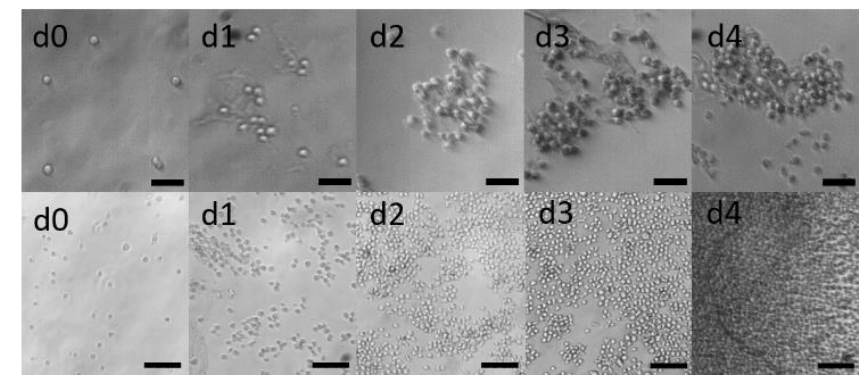

Figure 4. Viability and proliferation assays done for both Jurkat and C1R cells. a) Viability for different fluences at both working positions; b) proliferation of cells; upper row: C1R cells transferred at $2.1 \mathrm{~J} / \mathrm{cm}^{2}$; bottom row: Jurkat cells transferred at $5.7 \mathrm{~J} / \mathrm{cm}^{2}$. (All scale bars 100 $\mu m)$ 


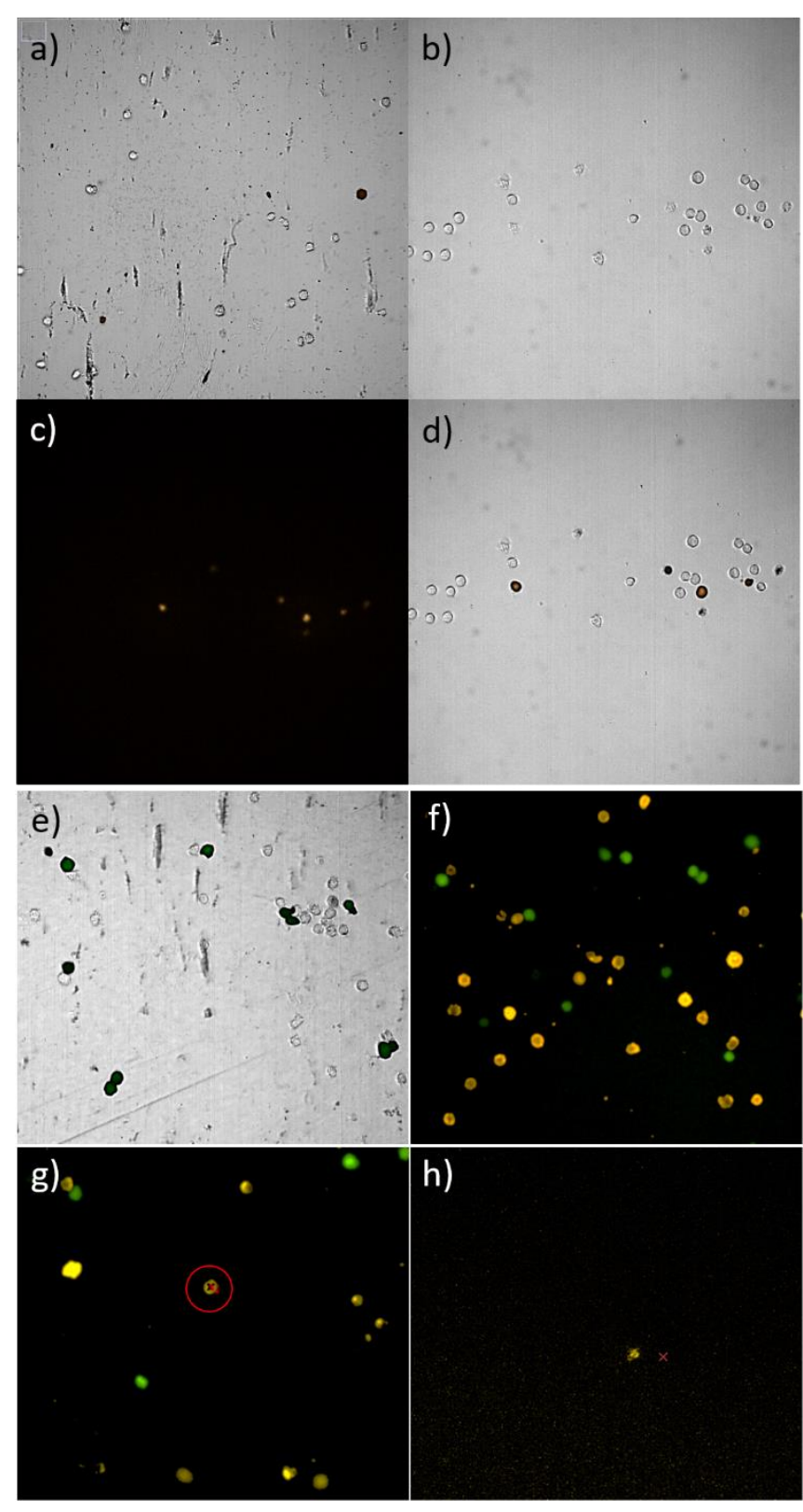

Figure 5. Cell viability and single cell detection and isolation using FE BA-LIFT. a) Superposed image of viable and dead cells in the donor substrate. b) Conventional microscopy image of transferred cells. c) Fluorescence image of transferred cells. d) Superposed imaged of transferred cells. All images are taken with the FE BALIFT system; e) Overlay image of both conventional microscopy and fluorescence of C1R cells expressing eGFP mixed with unlabeled Jurkat cells. f) Fluorescence image taken with the FE BA-LIFT system of C1R-eGFP cells mixed with Jurkat cells labelled with $\alpha-C D 45 / P E$. g) CD45+ cell, selected to transfer by the FE BA-LIFT system. $h$ ) Single transferred cell tracked with the same system used to select the cell on the donor.

First, C1R-eGFP cells were mixed with unlabeled Jurkat cells in a 1:1 ratio (Figure 4e); then C1R-eGFP cells were mixed with $\alpha$-CD45/PE labelled Jurkat cells (Figure 4f). All cells can be identified within the focal depth of the microscope objective; immediately after placing the donor-acceptor setup cells tend to deposit on the lower layer of the hydrogel. In both cases cells can be easily targeted, providing a suitable tool for the identification of specific cell lines within a complex sample. Furthermore, the capability of our LIFT system to isolate a single cell within a population of mixed cells was tested. Moreover, experiments showed that to isolate effectively a single cell a separation of minimum $50 \mu \mathrm{m}$ between cells was necessary, shorter distances result in transfer of more cells in the same droplet. The cell concentrations used were enough to keep almost all cells at least $50 \mu \mathrm{m}$ apart. Single cells can be isolated along the whole donor surface and cells are kept in good conditions during the whole experiment thanks to the sealed system designed that keeps the humid environment.

CD45+ Jurkat cell were selected (Figure $5 \mathrm{~g}$ ) and transferred to the receiving substrate (Figure 5h); cells survived the printing process and retained its membrane integrity, allowing to track them along the receiving substrate.

\section{NK cell transfer and activation assays}

To test whether the functionality of the cells is affected after the transfer process, a Natural Killer (NK) cell activation assay was performed. A NK cell line with a bi-cistronic expression of eGFP and IFN- $\gamma$ (Fontela et al., unpublished data) was transferred directly into stimulation culture medium (50ng PMA and $1 \mu \mathrm{g}$ ionomycin) and the amount of activation was measured as eGFP fluorescence which is similar to the IFN- $\gamma$ production (Figure 6). Fluorescence signal was observed after 4 hours (Figure 6d) and 6 hours (Figure 6f) being the most intense after 6 hours. A transferred single NK cell in bright and fluorescence field is shown in Figure 6a and $6 \mathrm{~b}$ respectively. PMA/Io activation favors cell aggregation; when transferring group of cells this phenomenon is observed, therefore indicating the laser transfer process does not affect NK cells metabolic activity and activation capabilities.

\section{HSPC's capacity of proliferation and formation of colony units (CFU)}

In order to assess the influence of the transfer process in the differentiation capacity of primary cells, transference of HSPC's was performed into a $35 \mathrm{~mm}$ diameter petri dish filled with $2 \mathrm{ml}$ of methylcellulose at $1.27 \%$. CFU were observed at different time points up to 14 days. HSPCs were selected as those non stained with antibodies specific for molecular markers of differentiated hematopoietic cells (Figure 7). On the Figure $7 \mathrm{c}$ it is shown the merged picture of the bright (Figure 7a) and fluorescent (Figure 7b) images; the cell marked with a circle was the HSPC selected for transfer. Figure $7 \mathrm{~d}$, e and $\mathrm{f}$ show the progression of colony formation from HSPC's transferred. The HSPC's after 14 days are shown in Figure 7g, 7h and 7i showing an amplified view of three distinct developed colonies. In this example, from a total 
60 HSPCs transferred, $55 \mathrm{CFU}$ were detected when considering colonies of 10 or more cells but as high as 80 when considering colonies of more than 5 cells. Therefore, we kept $100 \%$ of the proliferation and differentiation potential of the transferred HSPCs.

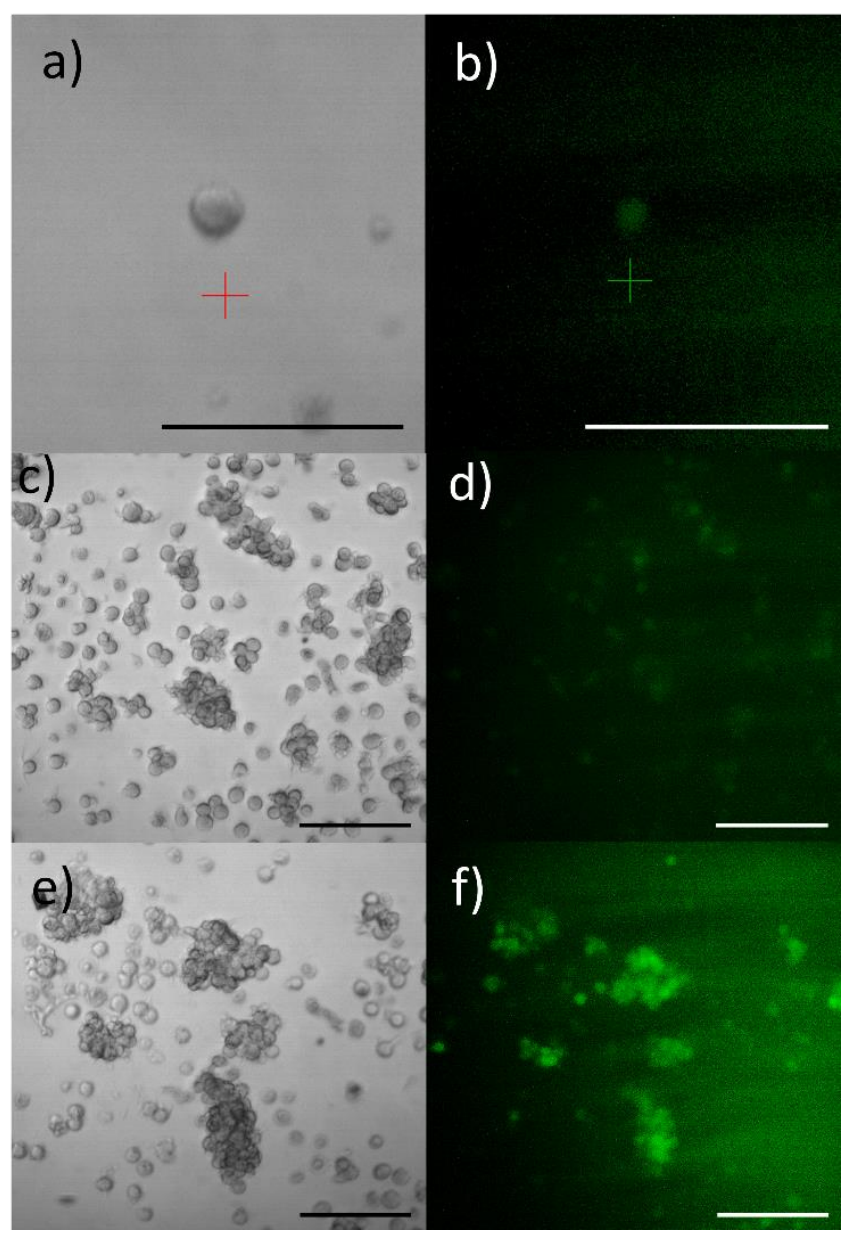

Figure 6. NK cells activation tracked through fluorescence. Single NK cell transferred in 96 well plate after 4-hour incubation a) bright field image and b) fluorescence image; groups of NK cells c) bright field image and d) fluorescence image after 4-hour incubation; e) bright field image and f) fluorescence image after 6-hour incubation. (All scale bars $100 \mu \mathrm{m}$ ) (Incubation done at $37^{\circ} \mathrm{C}$ and 5 $\% \mathrm{CO}_{2}$ environment) a)

d)
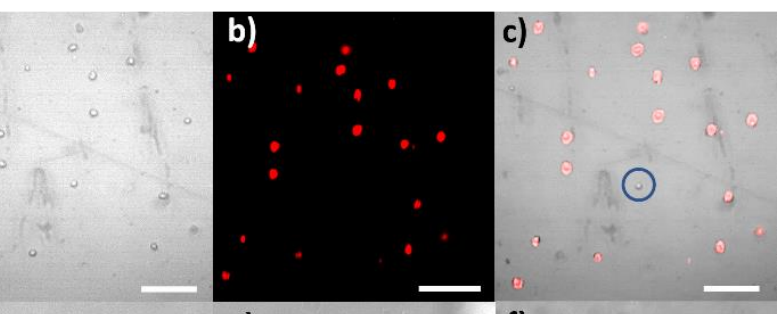

e)

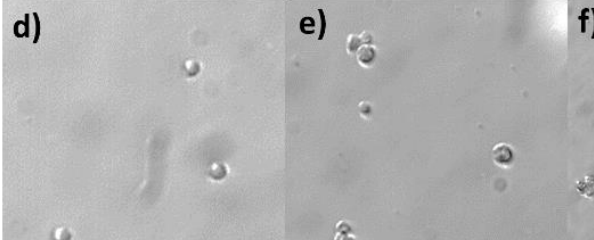

5

f)

6

g)
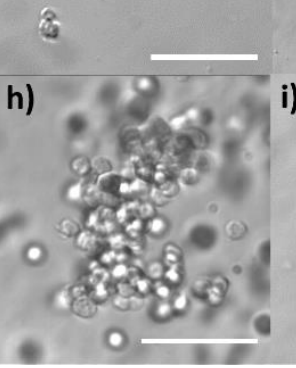

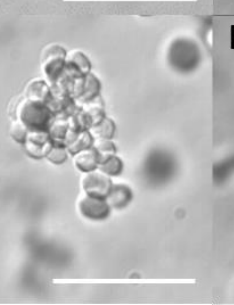

Figure 7. Bone marrow stem and progenitor cells selection and colonies development. Merged (c) of bright (a) and fluorescence (b) image of cells on the donor substrate. Culture of selected HSPCS at 1 day (d), 4 days (e) and 8 days (f); g), h) and i) close view of colonies after 14 days. (All scale bars $50 \mu \mathrm{m}$ )

\section{Discussion.}

A new approach for BA-LIFT technique has been adapted to print two different hydrogels, leading to a complete printability map. The use of an intermediate layer consisting of a commercial polyimide tape makes this process more accessible and easier to implement. Polyimide layer has shown two main benefits over the commonly used in LIFT: its absorption properties protect the living cells from laser radiation, and its transparency to visible light allows the use an in-line vision system with both conventional microscopy and fluorescence imaging, coaxial with the laser path. This set-up gives the possibility to measure cell viability before and after the LIFT process, applying an easy and non-toxic method to track cell survival in-situ. Additionally, the most promising application of our FE BA-LIFT system is the possibility to choose and transfer cells previously labelled with fluorescent surface markers, so that individual cells can be isolated from complex populations based on specific cell markers. These improvements make our approach better option than the commonly used in BA-LIFT technique [17].

Although BA-LIFT has already been performed with a polyimide layer acting as intermediate layer [17,18], a new approach using a commercial polyimide in form of adhesive tape is presented. The main advantages for using a commercially available polyimide tape are, on firsthand, the accessibility and ease to manufacture uniform polyimide coated microscope slides. Although spin coating is a very 
reliable process it takes a long time to prepare each sample and a lot of waste material is produced. Furthermore, polyimide is mechanically and chemically stable at a wide range of temperatures assuring that it won't degrade or release undesirable elements to the hydrogel.

It is important, in order to preserve the transferred material and the living cells, to assure that no direct laser irradiation or heating reach the biological sample [36,37]. In this approach and due to the very large UV absorption of the polyimide layer and its thickness $(\sim 30 \mu \mathrm{m})$ laser irradiation is completely absorbed within the first micron of the tape. Besides, thanks to the thermal properties of the material, there is no significant heating in the liquid layer avoiding thermal effects on the biological samples.

Several studies show that transfer using metallic layer is produced by complex hydrodynamical effects $[38,39]$. In this approach, the vaporization of the metallic layer leads to a cavitation bubble generation which quickly expands and releases a small amount of liquid [40-42]. With this approach, a certain heating of the liquid is unavoidable, together with some diffusion of metallic particles in the liquid, although good cell viability can be achieved [37,43-45].

Also, it was important to generate a printability map for this new BA-LIFT configuration for a wide range of hydrogel concentrations and set a range of parameters for the best transfer results. Previous works use a spin coated polyimide layer of $7 \mu \mathrm{m}$, one order of magnitude less than the commercial tape $(\sim 30 \mu \mathrm{m})$. Results show that transfer is possible for a wide range of energies and hydrogel viscosities even though the polyimide thickness is significantly thicker. Several studies of alginate and methylcellulose laser bioprinting have been made, mostly for sodium alginate [6,46-49]. This part of the study aims to widen the printability study of other authors and present it as a simple summary table.

Taking advantage of the transparency of polyimide to visible light a coaxial vision system to the laser was installed. Previous works done were made using a single standard microscopy camera to identify cells $[6,14,15,37,43,50-53]$. The proposed system integrates also a fluorescence detector allowing the system to discriminate cells not only by its shape and size but also by fluorescence markers attached to them. This is relevant to increase the specificity of cell detection, where depending on the complexity of the fluorescence system up to 12 sub populations of cells could be detected. This can be achieved by adding more filter sets to the system and more illumination sources so more fluorophores can be detected. Applications regarding single cell detecting and analysis could use the added value of having a live and instant cell isolation system. Also, by using a simple live/dead staining kit, a quick and reliable method for discriminating dead cells in-situ from both the donor and acceptor substrates is added. During a sorting procedure all dead cells from the sample could be ignored leaving only the viable cells for study.

The ability to trace NK cell activation within a PMA solution at a single cell level proves that cells keep their metabolic and signaling pathways. This study paves the way for future analysis of cell activation in a more complex environment like cell-cell interaction in an immunological model. The fact that the stems cells transferred with our LIFT system can proliferate and form colonies validates the technique to handle very sensitive primary cell types. Moreover, given the capability to isolate and track individual cells makes this system a suitable tool for single stem cell biology analysis such as, cell lineage tracking, time lapse imaging and single cell molecular profiling (proteomics, single cell genome sequencing and transcriptome sequencing and single cell RNA fish) [54].

\section{Conclusions}

A complete study for a new BA-LIFT configuration is presented, using a polyimide tape as energy absorption layer, therefore making it easier and cheaper to prepare the samples used as donor substrates. This study demonstrates the technique is feasible for cell laden hydrogel printing at a wide range of viscosities and droplet sizes. Moreover, the use of a semi-transparent layer as energy absorption layer provides the ability of enhancing the system with a fluorescence and conventional microscopy system used to track and select cells in both the donor and acceptor substrates. The technique proves to be a feasible tool for live tracking and isolation of single cells. Furthermore, the capability to detect and transfer a minor cell population from a complex group of primary cells was demonstrated, maintaining their pluripotency, differentiation and proliferation capacities.

\section{Acknowledgements}

Partial financial support by the Spanish Ministry of Science and Innovation under CHENOC (ENE2016-78933C4-4-R), Instituto de Salud Carlos III MPY 1346/16 and the Ministry of Education and Research of the Community of Madrid (BIOPIELTEC-CM-S2018/BAA-4480).

Author Miguel Gomez Fontela is grateful for the funding of the FPU grant given by the Spanish MECD, which allows the realization and publication of this work.

\section{References}

[1] Murphy S V and Atala A 2014 3D bioprinting of tissues and organs Nat. Biotechnol. 32 773-85

[2] Michael S, Sorg H, Peck C-T, Koch L, Deiwick A Chichkov B, Vogt P M and Reimers K 2013 Tissue Engineered Skin Substitutes Created by Laser-Assisted Bioprinting Form Skin-Like Structures in the Dorsal Skin Fold Chamber in Mice ed A T Slominski PLoS One 8 e57741

[3] Catros S, Guillemot F, Nandakumar A, Ziane S, Moroni L, Habibovic P, van Blitterswijk C, Rousseau B, 
Chassande O, Amédée J and Fricain J-C 2012 Layer-byLayer Tissue Microfabrication Supports Cell Proliferation In Vitro and In Vivo Tissue Eng. Part C Methods 18 62-70

[4] Chen C Y, Barron J A and Ringeisen B R 2006 Cell patterning without chemical surface modification: Cellcell interactions between printed bovine aortic endothelial cells (BAEC) on a homogeneous cell-adherent hydrogel Appl. Surf. Sci. 252 8641-5

[5] Othon C M, Wu X, Anders J J and Ringeisen B R 2008 Single-cell printing to form three-dimensional lines of olfactory ensheathing cells Biomed. Mater. 3034101

[6] Schiele N R, Corr D T, Huang Y, Raof N A, Xie Y and Chrisey D B 2010 Laser-based direct-write techniques for cell printing Biofabrication 2032001

[7] Ovsianikov A, Gruene M, Pflaum M, Koch L, Maiorana F, Wilhelmi M, Haverich A and Chichkov B 2010 Laser printing of cells into 3D scaffolds Biofabrication 2014104

[8] Gruene M, Pflaum M, Deiwick A, Koch L, Schlie S, Unger C, Wilhelmi M, Haverich A and Chichkov B N 2011 Adipogenic differentiation of laser-printed 3D tissue grafts consisting of human adipose-derived stem cells Biofabrication 3015005

[9] Barron J A, Wu P, Ladouceur H D and Ringeisen B R 2004 Biological Laser Printing: A Novel Technique for Creating Heterogeneous 3-dimensional Cell Patterns Biomed. Microdevices 6 139-47

[10] Catros S, Fricain J-C, Guillotin B, Pippenger B, Bareille R, Remy M, Lebraud E, Desbat B, Amédée J and Guillemot F 2011 Laser-assisted bioprinting for creating on-demand patterns of human osteoprogenitor cells and nano-hydroxyapatite Biofabrication 3025001

[11] Bourget J-M, Kérourédan O, Medina M, Rémy M, Thébaud N B, Bareille R, Chassande O, Amédée J, Catros S and Devillard R 2016 Patterning of Endothelial Cells and Mesenchymal Stem Cells by Laser-Assisted Bioprinting to Study Cell Migration Biomed Res. Int. 2016 1-7

[12] Vinson B T, Phamduy T B, Shipman J, Riggs B, Strong A L, Sklare S C, Murfee W L, Burow M E, Bunnell B A, Huang Y and Chrisey D B 2017 Laser direct-write based fabrication of a spatially-defined, biomimetic construct as a potential model for breast cancer cell invasion into adipose tissue Biofabrication 9

[13] Koch L, Deiwick A, Franke A, Schwanke K, Haverich A, Zweigerdt R and Chichkov B N 2018 Laser bioprinting of human induced pluripotent stem cells - the effect of printing and biomaterials on cell survival, pluripotency, and differentiation Biofabrication

[14] Kingsley D M, Dias a D, Chrisey D B and Corr D T 2013 Single-step laser-based fabrication and patterning of cellencapsulated alginate microbeads Biofabrication 5045006

[15] Dias a D, Unser a M, Xie Y, Chrisey D B and Corr D T 2014 Generating size-controlled embryoid bodies using laser direct-write Biofabrication 6025007

[16] Curley J L, Sklare S C, Bowser D A, Saksena J, Moore M J and Chrisey D B 2016 Isolated node engineering of neuronal systems using laser direct write Biofabrication $\mathbf{8}$ 015013

[17] Kattamis N T, Purnick P E, Weiss R and Arnold C B 2007 Thick film laser induced forward transfer for deposition of thermally and mechanically sensitive materials Appl. Phys. Lett. 91171120

[18] Brown M S, Kattamis N T and Arnold C B 2010 Timeresolved study of polyimide absorption layers for blisteractuated laser-induced forward transfer J. Appl. Phys. 107
$1-8$

[19] Turkoz E, Perazzo A, Kim H, Stone H A and Arnold C B 2018 Impulsively Induced Jets from Viscoelastic Films for High-Resolution Printing Phys. Rev. Lett. 12074501

[20] Turkoz E, Deike L and Arnold C B 2017 Comparison of jets from Newtonian and non-Newtonian fluids induced by blister-actuated laser-induced forward transfer (BA-LIFT) Appl. Phys. A Mater. Sci. Process. 123

[21] Brasz C F, Yang J H and Arnold C B 2014 Tilting of adjacent laser-induced liquid jets Microfluid. Nanofluidics 18 185-97

[22] Kattamis N T, Brown M S and Arnold C B 2011 Finite element analysis of blister formation in laser-induced forward transfer J. Mater. Res. 26 2438-49

[23] Bryant R G, Ryant R O G B and Langley N 2014 Polyimides Ullmann's Encyclopedia of Industrial Chemistry (American Cancer Society) pp 1-27

[24] Julien S, Peters T, Ziemssen F, Arango-Gonzalez B, Beck S, Thielecke H, Büth H, Van Vlierberghe S, Sirova M, Rossmann P, Rihova B, Schacht E, Dubruel P, Zrenner E and Schraermeyer U 2011 Implantation of ultrathin, biofunctionalized polyimide membranes into the subretinal space of rats Biomaterials 32 3890-8

[25] Herth E, Guerchouche K, Rousseau L, Calvet L E and Loyez C 2017 A biocompatible and flexible polyimide for wireless sensors Microsyst. Technol. 23 5921-9

[26] Myllymaa S, Myllymaa K, Korhonen H, Lammi M J, Tiitu V and Lappalainen R 2010 Surface characterization and in vitro biocompatibility assessment of photosensitive polyimide films Colloids Surfaces B Biointerfaces 76505 11

[27] Del Valle J, De La Oliva N, Müller M, Stieglitz T and Navarro X 2015 Biocompatibility evaluation of parylene C and polyimide as substrates for peripheral nerve interfaces Int. IEEE/EMBS Conf. Neural Eng. NER 2015-July 442-5

[28] Richardson R R, Miller J A and Reichert W M 1993 Polyimides as biomaterials: preliminary biocompatibility testing Biomaterials 14 627-35

[29] Starr P, Agrawal C M and Bailey S 2016 Biocompatibility of common polyimides with human endothelial cells for a cardiovascular microsensor J. Biomed. Mater. Res. Part A 104 406-12

[30] Combs C A and Shroff H 2017 Fluorescence Microscopy: A Concise Guide to Current Imaging Methods Current Protocols in Neuroscience vol 2017 (Hoboken, NJ, USA: John Wiley \& Sons, Inc.) pp 2.1.1-2.1.25

[31] Chambers D C, Carew A M, Lukowski S W and Powell J E 2019 Transcriptomics and single-cell RNA-sequencing Respirology 24 29-36

[32] Liu Y, Chen X, Zhang Y and Liu J 2019 Advancing single-cell proteomics and metabolomics with microfluidic technologies Analyst 144 846-58

[33] Gross A, Schoendube J, Zimmermann S, Steeb M, Zengerle R and Koltay P 2015 Technologies for SingleCell Isolation Int. J. Mol. Sci. 16 16897-919

[34] Brown M S, Kattamis N T and Arnold C B 2011 Timeresolved dynamics of laser-induced micro-jets from thin liquid films Microfluid. Nanofluidics 11 199-207

[35] Khalil S and Sun W 2009 Bioprinting endothelial cells with alginate for 3D tissue constructs. J. Biomech. Eng. 131111002

[36] Karu T 1999 Primary and secondary mechanisms of action of visible to near-IR radiation on cells $J$. Photochem. Photobiol. B Biol. 49 1-17 
[37] Deng Y, Renaud P, Guo Z, Huang Z and Chen Y 2017 Single cell isolation process with laser induced forward transfer J. Biol. Eng. 11 1-13

[38] Unger C, Gruene M, Koch L, Koch J and Chichkov B N 2011 Time-resolved imaging of hydrogel printing via laser-induced forward transfer Appl. Phys. A 103 271-7

[39] Biver E, Rapp L, Alloncle A-P, Serra P and Delaporte P 2014 High-speed multi-jets printing using laser forward transfer: Time-resolved study of the ejection dynamics Opt. Express 22 17122-34

[40] Mézel C, Hallo L, Souquet A, Breil J, Hébert D and Guillemot F 2009 Self-consistent modeling of jet formation process in the nanosecond laser pulse regime Phys. Plasmas 16123112

[41] Xiong R, Zhang Z, Shen J, Lin Y, Huang Y and Chrisey D B 2014 Bubble Formation Modeling During Laser Direct Writing of Glycerol Solutions J. Micro NanoManufacturing 3011004

[42] Patrascioiu a., Fernández-Pradas J M, Palla-Papavlu A, Morenza J L and Serra P 2014 Laser-generated liquid microjets: correlation between bubble dynamics and liquid ejection Microfluid. Nanofluidics 16 55-63

[43] Barron J a., Krizman D B and Ringeisen B R 2005 Laser Printing of Single Cells: Statistical Analysis, Cell Viability, and Stress Ann. Biomed. Eng. 33 121-30

[44] Ali M, Pages E, Ducom A, Fontaine A and Guillemot F 2014 Controlling laser-induced jet formation for bioprinting mesenchymal stem cells with high viability and high resolution Biofabrication 6045001

[45] Sorkio A, Koch L, Koivusalo L, Deiwick A, Miettinen S, Chichkov B and Skottman H 2018 Human stem cell based corneal tissue mimicking structures using laser-assisted 3D bioprinting and functional bioinks Biomaterials 171 57-71

[46] Fardel R, Nagel M, Nüesch F, Lippert T and Wokaun A 2007 Laser forward transfer using a sacrificial layer: Influence of the material properties Appl. Surf. Sci. 254 1322-6

[47] Gudapati H, Yan J, Huang Y and Chrisey D B 2014 Alginate gelation-induced cell death during laser-assisted cell printing Biofabrication 6035022

[48] Yan J, Huang Y and Chrisey D B 2013 Laser-assisted printing of alginate long tubes and annular constructs Biofabrication 5015002

[49] Zhang Z, Xiong R, Mei R, Huang Y and Chrisey D B 2015 Time-Resolved Imaging Study of Jetting Dynamics during Laser Printing of Viscoelastic Alginate Solutions Langmuir 31 6447-56

[50] Schiele N R, Koppes R a., Corr D T, Ellison K S, Thompson D M, Ligon L a., Lippert T K M and Chrisey D B 2009 Laser direct writing of combinatorial libraries of idealized cellular constructs: Biomedical applications Appl. Surf. Sci. 255 5444-7

[51] Xiong R, Zhang Z, Chai W, Chrisey D B and Huang Y 2017 Study of gelatin as an effective energy absorbing layer for laser bioprinting Biofabrication 9

[52] Phamduy T B, Raof N A, Schiele N R, Yan Z, Corr D T, Huang Y, Xie Y and Chrisey D B 2012 Laser direct-write of single microbeads into spatially-ordered patterns Biofabrication 4025006

[53] Riester D, Özmert A and Wehner M 2014 Laser tool for single cell transfer J. Laser Micro Nanoeng. 9 93-7

[54] Etzrodt M, Endele M and Schroeder T 2014 Quantitative single-cell approaches to stem cell research Cell Stem Cell 15 546-58 